\title{
NOTE ON FORMULAS FOR THE NUMBER OF REPRESENTATIONS OF AN INTEGER AS A SUM OF $2 h$ SQUARES*
}

BY R. D. JAMES

1. Introduction. It is known that the number of representations of an arbitrary integer $n$ as a sum of $2 h$ squares may be expressed in the form

$$
\lambda \sum(-1)^{(h d-h) / 2} d^{h-1},
$$

when $1 \leqq h \leqq 4$. The number $\lambda$ may depend on the linear form of $n$ and the summation is over all odd divisors $d$ of $n$. For example, an odd integer can be represented as a sum of four squares in $8 \sum d$ ways, while an even integer can be represented in $24 \sum d$ ways. The question arises whether formulas of the type (1) hold when $h \geqq 5$. This question has been answered in the negative for $n=m$ or $n=2 m$, where $m$ is odd, by E. T. Bell $\dagger$ and in some cases for $n=2^{\alpha} m, \alpha \geqq 1$ by the author.t These results were obtained by the use of the theory of elliptic functions.

In this note we shall prove a necessary condition which does not require any elliptic function theory and which enables us to decide if a formula of the type (1) is possible or not. It is to be noticed that this method does not show when formulas of the type (1) are true but only when they are not true.

2. Necessary Condition for Formulas of Type (1). Let $n_{j}$ denote any one of a given class $K\left(n_{j}\right)$ of positive integers in which $n_{j}<n_{j+1}$. For example, we might take $n_{j}=4 j-3$ and then $K\left(n_{j}\right)$ would be the class of all positive integers which are $\equiv 1(\bmod 4)$. Let $N(n, 2 h)$ denote the number of representations of an integer $n$ as a sum of $2 h$ squares, both the arrangement of the squares and the signs of their square roots being relevant in counting the representations. Then we have the following result, which

* Presented to the Society, April 11, 1936.

$\dagger$ Journal für die reine und angewandte Mathematik, vol. 163 (1930), pp. 65-70; Journal of the London Mathematical Society, vol. 4 (1929), pp. 279285.

$\ddagger$ American Journal of Mathematics, vol. 58 (1936), pp. 536-544. 
is almost trivial, but is nevertheless sufficient to show the impossibility of formulas like (1) in a great number of cases.

THEOREM 1. In order that the formula

$$
N(n, 2 h)=\lambda \sum_{d \mid n}(-1)^{(h d-h) / 2} d^{h-1}
$$

should hold for every integer in the class $K\left(n_{j}\right)$ with $\lambda$ independent of $n$, it is necessary that

$$
\begin{aligned}
N\left(n_{2}, 2 h\right) \sum_{d \mid n_{1}}(-1)^{(h d-h) / 2} d^{h-1} & \\
= & N\left(n_{1}, 2 h\right) \sum_{d \mid n_{2}}(-1)^{(h d-h) / 2} d^{h-1} .
\end{aligned}
$$

The summations in each case are over all odd divisors of $n, n_{1}$, and $n_{2}$, respectively.

Proof. If the formula (2) is to hold for all $n$ in $K\left(n_{j}\right)$, it must certainly hold when $n=n_{1}$ and $n=n_{2}$. The necessary condition (3) is obtained by equating the values of $\lambda$ found for these two values of $n$.

3. Impossibility of Formulas of Type (2). We now give a few results to illustrate the use of Theorem 1.

THEOREM 2.* If $h \geqq 5$ no formula of the type (2) can hold for all odd integers $m \equiv 1(\bmod 4)$ with $\lambda$ independent of $m$.

Proof. Here we have $K\left(n_{j}\right)=K(4 j-3)$ and $n_{1}=1, n_{2}=5$. The condition (3) becomes

$$
N(5,2 h)=N(1,2 h)\left[1+5^{h-1}\right] .
$$

Since

$$
\begin{aligned}
& 1=( \pm 1)^{2}+0^{2}+\cdots+0^{2} \\
& 5=( \pm 1)^{2}+( \pm 2)^{2}+0^{2}+\cdots+0^{2} \\
& 5=( \pm 1)^{2}+\cdots+( \pm 1)^{2}+0^{2}+\cdots+0^{2}
\end{aligned}
$$

we find that $\dagger$

* E. T. Bell, loc. cit.

$\dagger C_{n, t}$ denotes the binomial coefficient $n ! / t !(n-t)$ ! 


$$
\begin{aligned}
& N(1,2 h)=2 C_{2 h, 1}, \\
& N(5,2 h)=2{ }^{2} C_{2 h, 1} \cdot C_{2 h-1,1}+2{ }^{5} C_{2 h, 5} .
\end{aligned}
$$

Substitution of these values in (4) reduces the condition to the form

$30(2 h-1)+2(2 h-1)(2 h-2)(2 h-3)(2 h-4)=15\left(1+5^{h-1}\right)$.

For $h=2 r+1$ this equation is identical with the one given by E. T. Bell in Theorem $\alpha$ of his Journal für Mathematik paper referred to above. It can be verified that this equation is true only when $1 \leqq h \leqq 4$. In fact, for $h \geqq 5$ the left side is less than the right side.

Theorem 3. If $h$ is odd and $\geqq 3$, or if $h$ is even and $\geqq 8$, no formulas of the type (2) can hold for all integers of the form $2 m, m$ odd, with $\lambda$ independent of $m$.

Proof. In this case we have $K\left(n_{j}\right)=K(4 j-2)$ and $n_{1}=2$, $n_{2}=6$. Then (3) becomes

$$
N(6,2 h)=N(2,2 h)\left[1+(-1)^{h} 3^{h-1}\right] .
$$

Since

$$
\begin{aligned}
& 2=( \pm 1)^{2}+( \pm 1)^{2}+0^{2}+\cdots+0^{2} \\
& 6=( \pm 1)^{2}+( \pm 1)^{2}+( \pm 2)^{2}+0^{2}+\cdots+0^{2} \\
& 6=( \pm 1)^{2}+\cdots+( \pm 1)^{2}+0^{2}+\cdots+0^{2}
\end{aligned}
$$

we have

$$
\begin{aligned}
& N(2,2 h)=2{ }^{2} C_{2 h, 2}, \\
& N(6,2 h)=2{ }^{3} C_{2 h, 2} \cdot C_{2 h-2,1}+2{ }^{6} C_{2 h, 6} .
\end{aligned}
$$

After simplification the condition becomes

$$
(4 h-4)[45+(2 h-3)(2 h-4)(2 h-5)]=45\left[1+(-1)^{h} 3^{h-1}\right] .
$$

This equation is true only when $h=1,2,4$, or 6 . For $h$ odd and $\geqq 3$ the left side is greater than the right side. For $h$ even and $\geqq 8$ the left side is less than the right side.

\footnotetext{
* E. T. Bell, loc. cit.
} 
THEOREM 4. If $h=5$ or $h \geqq 7$, no formulas of the type (2) can hold for all integers of the form $2 m, m \equiv 1(\bmod 4)$, with $\lambda$ independent of $m$.

Proof. This time we have $K\left(n_{j}\right)=K(8 j-6), n_{1}=2, n_{2}=10$. Then (3) becomes

$$
N(10,2 h)=N(2,2 h)\left[1+5^{h-1}\right] .
$$

Since

$$
\begin{aligned}
& 10=( \pm 1)^{2}+( \pm 3)^{2}+0^{2}+\cdots+0^{2}, \\
& 10=( \pm 1)^{2}+( \pm 1)^{2}+( \pm 2)^{2}+( \pm 2)^{2}+0^{2}+\cdots+0^{2}, \\
& 10=( \pm 1)^{2}+\cdots+( \pm 1)^{2}+( \pm 2)^{2}+0^{2}+\cdots+0^{2}, \\
& 10=( \pm 1)^{2}+\cdots+( \pm 1)^{2}+0^{2}+\cdots+0^{2}
\end{aligned}
$$

we have

$$
\begin{array}{r}
N(10,2 h)=2^{2} C_{2,1} \cdot C_{2 h, 2}+2{ }^{4} C_{2 h, 2} \cdot C_{2 h-2,2} \\
+2 C_{2 h, 6} \cdot C_{2 h-6,1}+2{ }^{10} C_{2 h, 10} .
\end{array}
$$

After simplification the condition reduces to

$$
\begin{array}{r}
28350[1+(2 h-2)(2 h-3)]+1260(2 h-2) \cdots(2 h-6) \\
+2(2 h-2) \cdots(2 h-9)=14175\left[1+5^{h-1}\right] .
\end{array}
$$

It can be verified that this equation is true for $1 \leqq h \leqq 6, h \neq 5$, but that the left side is less than the right side when $h \geqq 7$, and the right side is less than the left side when $h=5$.

The University of California 